\title{
VERDAD Y SUBJETIVIDAD EN EL POSTSCRIPTUM \\ DE S. KIERKEGAARD
}

\author{
Manuel Zelada
}

Pontificia Universidad Católica del Perú

Resumen: El siguiente texto ofrece un análisis del sentido del término verdad subjetiva para el pensamiento kierkegaardiano a partir de las reflexiones presentes en el Postscriptum no científico y definitivo a Migajas filosóficas. La intención de este trabajo es recoger las reflexiones sobre la objetividad y la subjetividad que Kierkegaard desarrolla en relación con la verdad subjetiva para mostrar que, lejos de afirmar que es la interioridad del sujeto la que determina la verdad (subjetivismo), esta implica siempre algo más allá del sujeto mismo.

Palabras clave: Kierkegaard, verdad, subjetividad, creencia, subjetivismo.

Abstract: The following commentary offers an analysis of subjective truth in Soren Kierkegaard's Concluding Unscientific Postscript to his Philosophical Fragments. Our intention is to discuss Kierkegaard's reflections on subjective truth, in order to show that, rather than claiming that the subject's interiority defines truth (subjectivism), Kierkegaard thought that truth supposes more than the subject itself.

Keywords: Kierkegaard, truth, subjectivity, belief, subjectivism. 
Quizás una de las frases más conocidas de Kierkegaard-si no la más conocidasea "La verdad es subjetividad" 349 . Adorno, entre otros lectores, creía ver en ella un riesgoso acercamiento al subjetivismo, a la vez que una confirmación de la cercanía entre Kierkegaard y el existencialismo ${ }^{350}$. Así vista, la frase, además de ubicarse en un discurso sobre la búsqueda de la autenticidad o el cuidado de la interioridad individual (temas, sin duda, presentes en Kierkegaard) podía interpretarse como una exacerbación del individuo. Sin embargo, pocas veces tal frase motivaba un acercamiento al problema del estatuto de la verdad, el cual se discute en las mismas páginas del Postscriptum de las cuales suele extrapolarse.

Por ello, quisiera reflexionar con ustedes sobre algunos temas que se abren en razón de la afirmación "la verdad es subjetividad". Pretender agotarlos sería excesivo; me conformo con dar algunas luces sobre el sentido de la frase y sobre la vigencia de las reflexiones en las que se articula. Para ello presentaré, primero, el contexto en el que se enuncia; luego, proseguiré presentado el problema del subjetivismo y una posible defensa presente en Kierkegaard. Finalmente, haré una pequeña síntesis sobre lo que podríamos entender en relación a la verdad subjetiva que defiende la citada frase.

"La verdad es subjetividad" es el nombre de un capítulo del Postscriptum no científico y definitivo a Migajas filosóficas. Cito el nombre completo porque este libro es, ciertamente, un postscriptum, un añadido, una apostilla a otro texto llamado Migajas filosóficas. Tanto en Migajas como en el Postscriptum, el tema central es la creencia (en particular, la creencia religiosa) y su relación con el creyente. Si, como decíamos, la frase citada se ubica en el marco de una reflexión sobre la verdad, esta reflexión tiene lugar, precisamente, para esclarecer las semejanzas y diferencias entre aquella verdad que se tiene por cierta en función a un argumento común y demostrable y aquella que se tiene por cierta en función de una fe o, parafraseando a James, una voluntad de creer. Es necesario tener esta tematización de la verdad sobre el trasfondo

349 Kierkegaard, Soren, Postscriptum no científico y definitivo a Migajas filosóficas, traducción de Nassim Bravo Jordán, México D.F.: Universidad Iberoamericana, 2009, p. 191.

350 Cf. Adorno, Theodor, Kierkegaard. Construcción de lo estético, traducción de Roberto Venergo, Caracas: Monte Ávila, 1969; cf. Adorno, Theodor, Dialéctica negativa, traducción de J. M. Ripalda, Madrid: Taurus, 1975, la primera sección; también cf. Kroner, Richard J., "iKierkegaard or Hegel?", en: Revue internationale de philosophie, No. 19 (1952), pp. 79-96. 
de la creencia para poder entender mejor el análisis de la verdad que hace Kierkegaard.

En primer lugar, distingue entre una verdad idealista, para la que el ser debe adecuarse al pensamiento, y una verdad empirista, para la cual el pensamiento debe adecuarse al ser. Nos encontramos, pues, ante la pregunta sobre si la verdad presupone una idea o un orden pensable anterior al objeto a conocer o si, por el contrario, debe fidelidad al aparecer concreto de ese objeto. La primera opción es vista por Kierkegaard como una tautología y es descartada, dado que supone que el ser es solo una ocasión que se configura y acomoda según el pensamiento. Sin embargo, la segunda debe soportar el hecho de que la condición empírica de su objeto sitúe la búsqueda de la verdad empírica al interior de la variabilidad y contingencia propias del mundo natural e histórico. Frente a la correspondencia necesaria entre pensamiento y ser propia de la verdad idealista, la verdad empírica no puede ofrecer sino una aproximación entre ambos. La opción kierkegaardiana por esta no se basa, por lo tanto, en un criterio formal de necesidad, sino en un privilegio de la concreción (tanto del sujeto cognoscente como del objeto) frente a lo abstracto del pensamiento ${ }^{351}$. Dicha opción, cabe decirlo, no supone que la verificabilidad de la existencia del sujeto o del objeto haya dejado de ser un problema; por el contrario, si ambos se hallan sometidos a la contingencia propia de la existencia concreta, entonces la posibilidad de conocimiento de ambos, así como la realidad de su existencia, no pueden ser objeto de un conocimiento, sino un principio establecido por una decisión en virtud de la fe $\mathrm{fe}^{352}$.

En otras palabras, el principio del cual parte la búsqueda de la verdad tiene su origen en una decisión subjetiva en virtud de una creencia subjetiva de tal modo que incluso la certeza de una verdad objetiva sostenida en datos verificables $y$ argumentos compartidos se sostiene, en principio, en una creencia subjetiva. Desde luego, Kierkegaard establece una distinción entre tal verdad objetiva y una creencia subjetiva (en adelante, verdad subjetiva), pero concibe, también, una necesaria relación entre ambas. La forma en la que hemos presentado esta relación (como una interdependencia) nos lleva a la siguiente pregunta:

351 Cf. Kierkegaard, op. cit., pp.191-196.

352 Cf. Kierkegaard, Soren, Migajas filosóficas o un poco de filosofía, traducción de Rafael Larrañeta, Madrid: Trotta, 1997, pp.86-92. 
iestá negando Kierkegaard la posibilidad de un criterio de verificación que permita distinguir lo verdadero de lo falso más allá del ámbito propio de cada individuo cognoscente? De responder afirmativamente tendríamos que lidiar con el subjetivismo, es decir, con el hecho de que el valor de nuestros juicios dependa únicamente de criterios subjetivos relativos al individuo que los enuncia ${ }^{353}$.

Afortunadamente, Kierkegaard es consciente de esta posible objeción. Lo manifiesta al exponer que uno de los riesgos comúnmente asociados a la defensa del carácter subjetivo de la verdad es la locura; esto es, la incapacidad de distinguir adecuadamente el pensamiento predicado a partir del ser. Esta absoluta ininteligibilidad del concepto, sin embargo, responde a un problema más amplio y más serio que la locura: el distanciamiento de un marco de inter-comprensión ${ }^{354}$. Ciertamente, si el criterio para determinar el valor de un juicio es meramente subjetivo, entonces nada exige ni garantiza la posibilidad de compartir dicho criterio, lo cual lleva a la imposibilidad de verificar la verdad o falsedad de un enunciado y, en última instancia, a la imposibilidad de comprender siquiera el sentido del enunciado.

Como defensa, Kierkegaard sostiene dos argumentos. El primero establece una distinción entre la locura y la verdad subjetiva. La primera se caracteriza por la insistencia obsesiva en una idea, mientras el contenido de la segunda es la infinitud misma. Para entender lo primero basta pensar en nuestra caracterización común del loco como alguien que sostiene algo en total desacuerdo con la realidad; en ese sentido, la locura se asemeja bastante a la verdad idealista, solo que a diferencia de esta, no siempre logra para sí misma adecuar el pensamiento al ser (pensemos, por ejemplo, en la dolorosa condición de la esquizofrenia o la depresión química). A diferencia de la creencia determinada y fija del loco, la verdad subjetiva se configura por la infinitud; es decir, no posee nunca un contenido determinado en relación con el ser, sino, por el contrario, se proyecta a un contenido que siempre está abierto a un nuevo aporte o una nueva reconfiguración. Podríamos decir, por ello,

353 Uno de los análisis más extensos en relación con este problema en el pensamiento de Kierkegaard lo ha realizado C.S. Evans (cf. Evans, C.S., "Kierkegaard on religious authority: the problem of the criterion" en: Faith and religión, VII, No. I (2000), pp. 48-67; también cf. Evans, C.S., Faith beyond reason. A kierkegaardian account, Edimburgo: Edinburgh University, 1998).

354 Cf. Kierkegaard, Postscriptum no científico y definitivo a Migajas filosóficas, p. 196. 
que frente a la frustrante imposibilidad de adecuar el pensamiento al ser de la locura, la verdad subjetiva se proyecta a la tarea infinita de adecuar el ser al pensamiento ${ }^{355}$.

Podríamos interpretar esta apertura a la infinitud como una creencia moral que se evidencia en una actitud para la cual el ser humano no puede ser tomado como un objeto de nuestras determinaciones; así, resultaría enriquecedor poner a la verdad subjetiva en diálogo con el hombre como fin en sí mismo kantiano, o la alteridad radical levinasiana. Podríamos, de otro modo, pensar esta apertura a la infinitud en relación con la tarea científica y reconocer, como James, que sin una creencia en la verdad que esté dispuesta a no darla nunca por sentada el avance científico sería imposible; que sin una verdadera fidelidad al objeto de estudio y su condición variable toda teoría caería en el dogmatismo 356 .

Retomando la defensa de Kierkegaard, diremos que el primer argumento hace énfasis en el contenido de la verdad subjetiva (la infinitud). El segundo, en cambio, enfatiza el modo de darse esta. Kierkegaard desarrolla este argumento a través de una historia: la anécdota del loco fugitivo. En ella, nuestro autor danés cuenta que una vez un loco se escapó del manicomio y mientras se preguntaba cómo hacer para que nadie note que estaba loco vio una bola de bolos y la colocó en el pliegue posterior de su saco de tal modo que al caminar rebotase contra su trasero. Esto le dio una idea: si caminaba afirmando una verdad tan evidente como que la tierra era redonda (como la bola) todos pensarían que debía estar cuerdo, dado que sus enunciados eran perfectamente verificables. Como podemos imaginar, la idea no dio resultado ${ }^{357}$.

Con esta anécdota, Kierkegaard pone de manifiesto que la sola objetividad de un juicio no basta para su adecuada evaluación, sino que es necesario que, además, sea puesto en relación con la existencia del sujeto cognoscente. Una forma preliminar de esclarecer esto es vinculando el concepto de existencia con el de marco interpretativo. Ciertamente, la existencia otorga un marco

355 Cf. ibid.

356 Cf. James, William, La voluntad de creer y otros ensayos de filosofía popular, traducción de Carmen Izco, Barcelona: Marbot, 2004, pp. II-47.

I66 357 Cf. Kierkegaard, Postscriptum no científico y definitivo a Migajas filosóficas, p. 197. 
interpretativo para nuestros juicios (en ella reconocemos quién lo dice, cómo lo dice, porqué lo dice), pero la existencia es un concepto mucho más amplio, supone también un marco de relevancia o utilidad, un ámbito de función, un modo de articulación y enunciación... Vincular un juicio con la existencia del sujeto cognoscente puede interpretarse entonces como identificar las situaciones en las cuales puede darse la mejor interpretación. Sin embargo, existe un sentido complementario para dicha afirmación y es que una valoración plena requiere de la coherencia entre el pensamiento y el actuar. Ciertamente, ni Kierkegaard ni nadie pretenderían buscarle un correlato práctico en la vida cotidiana de un astrónomo a alguna teoría suya, pero sí que su creencia en la verdad se halle lo suficientemente arraigada en él como para no tener que estar dudando todo el tiempo de la sinceridad de sus afirmaciones y pruebas. De hecho, debemos suponer que compartimos una creencia sincera en la verdad con un autor al momento de iniciarnos en cualquier teoría suya, más allá, incluso, de cuál sea el contenido de tal creencia. Ahora bien, para el astrónomo mismo, es posible suponer que su creencia en la verdad se pondrá el juego todo el tiempo que diseñe, exponga y cuestione (o cuestionen) su teoría, en la medida en que ella expresa un intento de aproximación a la verdad en la cual él cree.

Entonces, una verdad subjetiva o creencia se define en y por el propio actuar del individuo, en la medida en que este actuar se dé en situaciones en las cuales se ponga en juego dicha creencia. Por ello, el que la tierra sea redonda era algo absolutamente irrelevante tanto para la situación como el actuar del loco fugitivo $y$, entonces, no constituía ninguna verdad subjetiva.

Hemos analizado la validez y relevancia de este argumento para el caso de la ciencia, pero conviene pensar en otros. Por ejemplo, en lo pertinentes que serían sus conclusiones sobre la verdad para una época en la cual la distancia entre la vida profesional y la vida personal parece habernos llevado a una crisis de la que el estrés -con la cantidad de prácticas y escritos al respecto- parece ser su principal síntoma.

Hasta aquí no pretendo haberlos convencido de que Kierkegaard se haya libre de todo riesgo subjetivista, pero sí haber mostrado que, por lo menos, no era un ingenuo en relación con ello y que el planteamiento de la verdad subjetiva que subyace a la frase "la verdad es subjetividad" puede ser motivo 
de diversas reflexiones sobre ética, epistemología o simplemente el rol de la creencia en la vida cotidiana que exceden el ámbito del existencialismo y la filosofía de la religión, sin desmerecer su vigencia también en estos.

Resumiendo, quisiera destacar principalmente tres rasgos de la verdad subjetiva que han aparecido en esta reflexión. El primero, que no supone un rechazo de la verdad objetiva sino que, por el contrario, establece con ella una relación de interdependencia en la que se coloca como lo que podríamos llamar su fundamento volitivo y que, además, le permite situar a la verdad objetiva en relación con la existencia del cognoscente. El segundo, que la verdad subjetiva supone una relación entre existencia y pensamiento que debe verse en dos niveles: por un lado, implica el reconocimiento de la propia situación en la que uno se halla y a partir de la cual se actúa o enuncia; por otro lado, ( $y$ de especial relevancia para la verdad objetiva) implica la fidelidad al hecho concreto de la existencia del objeto y, por lo tanto, la atención a su variabilidad. Finalmente, el tercer rasgo es que la verdad subjetiva se juega en y por la existencia misma: en la existencia, porque el actuar del individuo configura y expresa el contenido de la creencia; y por la existencia, porque es el modo de existencia mismo del individuo, en sus diferentes situaciones y aspectos, el que se ve comprometido en dicho actuar. 